\title{
Population estimates of Endangered Mongolian saiga Saiga tatarica mongolica: implications for effective monitoring and population recovery
}

\author{
Julie K. Young, Kim M. Murray, Samantha Strindberg \\ BAYARB A A T R BUUVEIB A T A R and JOEL BERGER
}

\begin{abstract}
The global population of saiga Saiga tatarica, categorized as Critically Endangered on the IUCN Red List, declined by $>95 \%$ at the end of the 20th century, resulting in several conservation initiatives to protect the species. Previously used methods to monitor population trends were inadequate to assess numbers of saiga properly. We report findings from the first survey for Mongolian saiga S. tatarica mongolica to utilize statistically rigorous methodology, using line transect distance sampling in 2006 and 2007 to obtain population estimates in and around the Sharga Nature Reserve, the southern part of the species' current range. We estimate a density of 0.54 and 0.78 saiga $\mathrm{km}^{-2}$ in 2006 and 2007 , respectively. Our best models suggest that 4,938 (95\% confidence interval, $\mathrm{CI}=2,762-8,828)$ saiga occupied the $4,524-\mathrm{km}^{2}$ study area in 2006 and 7,221 (95\% CI = 4,38011,903 ) occupied the $4,678-\mathrm{km}^{2}$ study area in 2007. Although these estimates, with their large confidence intervals, preclude an assessment of the impacts of conservation initiatives on population trends, they suggest that the Mongolian saiga population is larger than previous reports based on minimum counts, and adequate to support in situ population recovery. Modifications to the survey protocol hold promise for improving the precision of future estimates. Distance sampling may be a useful, scientifically defensible method for monitoring saiga population trends and assessing the effectiveness of conservation efforts to stabilize and recover populations.
\end{abstract}

Keywords Antelope, distance sampling, endangered species, line transects, Mongolia, population estimate, Saiga tatarica

Julie K. YounG* (Corresponding author), Kim M. MurraY ${ }^{\dagger}$ and Joel BERGER $^{\ddagger}$ Wildlife Conservation Society, Northern Rockies Field Office, University of Montana, Missoula, Montana, USA. E-mail young@iws.org

Samantha Strindberg Wildlife Conservation Society, New York, USA

BayarbaAtar BuUveibaatar Institute of Biology, Mongolian Academy of Sciences, Ulaanbaatar, Mongolia

${ }^{*}$ Current address: Institute for Wildlife Studies, PO Box 1104, Arcata, California, USA

${ }^{\dagger}$ Current address: Snow Leopard Trust, Seattle, Washington, USA

${ }^{\ddagger}$ Also at: Division of Biological Science, University of Montana, Missoula, Montana, USA

Received 8 April 2009. Revision requested 29 April 2009.

Accepted 11 June 2009.

\section{Introduction}

Tstimates of population size are vital for understanding $\beth_{\text {species ecology, providing information on fluctuations }}$ in size and enabling monitoring of population trends. For threatened species population estimates are crucial for developing conservation strategies and assessing their effectiveness but obtaining estimates of species that occur at low numbers and inhabit large geographical areas is logistically difficult. Consequently, information on population size is often lacking for such species.

Saiga Saiga tatarica is a nomadic, sexually dimorphic species that was formerly widespread across the Central Asian steppe (Bekenov et al., 1998; Schaller, 1998). S. tatarica tatarica occurs in Kazakhstan, Russia, Uzbekistan and Turkmenistan, and Mongolian saiga S. tatarica mongolica, categorized as Endangered on the IUCN Red List (Mallon, 2008), is only found in Mongolia. The Mongolian subspecies is ecologically, phenotypically and behaviourally distinct (Bannikov et al., 1961; Kholodova et al., 2001). Because of overharvesting, saiga suffered a global population decline of c. $95 \%$ in $<_{15}$ years (Milner-Gulland et al., 2001). This severe decline led to the signing of a Memorandum of Understanding (MOU) among range states, under the Convention of Migratory Species (CMS, 2006), which established the need to adopt a standardized monitoring protocol to assess population numbers regularly. The MOU emphasized the need to evaluate the impact of natural and human-induced threats on saiga populations. Although the Mongolian saiga is not officially included in the MOU, the decline and resulting CMS raised awareness of the paucity of information on the population status and distribution of this subspecies (Lushchekina et al., 1999). Varied but consistent counts suggest that $<5,000$ Mongolian saiga remain in the wild (Clark \& Javzansuren, 2006; Chimeddorj et al., 2009).

Methods used previously for estimating population sizes of Mongolian saiga provided only a measure of relative abundance, with no corresponding measure of uncertainty, precluding statistical comparisons (Chimeddorj et al., 2009). An absolute estimate of abundance, and its associated estimate of variance, is required to assess and monitor population size properly. Methods should be accurate, repeatable and statistically rigorous. We therefore used 
distance sampling (Buckland et al., 2001) because it is a well-developed methodology that has been successfully applied to many ungulate species (Biswas \& Sankar, 2002; Koenen et al., 2002; Seddon et al., 2003; Whittaker et al., 2003; Focardi et al., 2005). Distance sampling methods are flexible and efficient for sampling sparse populations distributed over large regions (Olson et al., 2005), factors important for adoption of a technique to estimate population size throughout saiga range states.

In line-transect distance sampling observers traverse a series of transects and record perpendicular distance to detected groups. The group is used as the unit of observation when there is dependence among individuals in species that aggregate, and group size is recorded. Radial distance and angle are measured to calculate the perpendicular distance between the transect line and centre of the group. The probability of detecting a group is modelled as a function of the observed perpendicular distances and then combined with the estimated group encounter rate and estimated expected group size to calculate the density of individuals in the study area; abundance is also calculated if the total area of the study region is known (Buckland et al., 2001). Estimating the probability of detecting a group corrects for the number of animals undetected and provides absolute density and abundance estimates. Estimates are considered valid if four assumptions are met: (1) all individuals or groups on the transect line are detected with certainty, (2) animals are detected at their original location, (3) measurements are exact, and (4) transects are randomly placed with respect to animal or group distribution (Buckland et al., 2001).

We report population estimates of Mongolian saiga based on 2 years of distance sampling surveys, and discuss implications for in situ recovery of Mongolian saiga. We discuss the challenges in meeting the first two assumptions for estimating population density of saiga and suggest modifications to survey methodology that could improve the precision of estimates, making the technique suitable for range-wide adoption as a standardized monitoring protocol.

\section{Study area}

The study was conducted in and around the Sharga Nature Reserve (Gobi-Altai Aimag) in western Mongolia (Fig. 1). Clark \& Javzansuren (2006) suggest that c. 90\% of the Mongolian saiga population occurs within or near the Reserve. Our study area included c. $35 \%$ of the current range of Mongolian saiga (L. Amgalan, pers. comm.). Mean total annual precipitation is c. $50 \mathrm{~mm} \mathrm{year}^{-1}$, with much of it from winter snow. Annual temperatures are -30 to $+30^{\circ} \mathrm{C}$. The area is semi-desert and the predominant vegetation includes Allium, Stipa, Anabasis and Salsola. The only other ungulate to occupy the area is goitered gazelle Gazella subgutturosa. Altitudes are 1,300-2,100 m.

\section{Methods}

We established 24 15-km transects (Fig. 1), placed systematically to provide complete coverage of the study area. Although the starting point for the first transect was not selected randomly it was chosen without prior information regarding habitat, terrain, or other ecological characteristics. Cardinal directions for travel were selected so that transects traversed the numerous ravines that extended along the slopes of the valley because saiga often used these ravines as cover from wind and sun when resting during the day. Transects were spaced $\geq 5 \mathrm{~km}$ apart to avoid double counting, which could occur if saiga were displaced by observers from one transect to the next. Distance sampling was conducted in $<2$ weeks in September 2006 and 2007, with 1-5 transects completed each day. Most observers were different between the two surveys. Some transects could not be completed in their entirety because of difficult terrain and mechanical failures of the vehicles. Therefore, the length of transects varied slightly between years and two of the transects were not used in 2006. This resulted in differences in sampling effort and size of the study area between years (Table 1).

Transects were driven during daylight hours using a global positioning system for orientation. When saiga were detected group size, radial distance $(r)$ and sighting angle $(\theta)$ were recorded, using a compass, binoculars, spotting scope and rangefinder. Saiga often began to run after we detected them and, in these cases, we used a landscape feature at the point of detection to measure $r$ and $\theta$. From these data we calculated perpendicular distance as $x=r \sin (\theta)$. Density of saiga groups within the area surveyed $\left(D_{g}\right)$ was then estimated as:

$$
\hat{D}_{g}=\frac{\mathrm{n} \hat{f}(0)}{2 L}
$$

where $L$ denotes the aggregate length of the transects, $n$ is the number of saiga groups observed and $f(0)$ is the probability density function of observed perpendicular distances evaluated at $x=0$ (Buckland et al., 2001). Thus, density estimates are obtained from estimates of $f(0)$ and encounter rate $(\mathrm{n} / L) . f(0)$ is equal to $1 / \mu$, where $\mu$ is the effective strip half-width, corresponding to the perpendicular distance from the transect line within which the number of undetected groups is equal to the number of groups detected beyond it. Multiplying double the effective strip half-width by the aggregate length of the transects yields the effective area surveyed. Saiga density $(D)$ is obtained by multiplying the estimated group density by the estimated expected group size $\hat{E}(s)$. The density of individuals is multiplied by the surface area of the study area or survey stratum to obtain the corresponding abundance estimate $(N)$. 


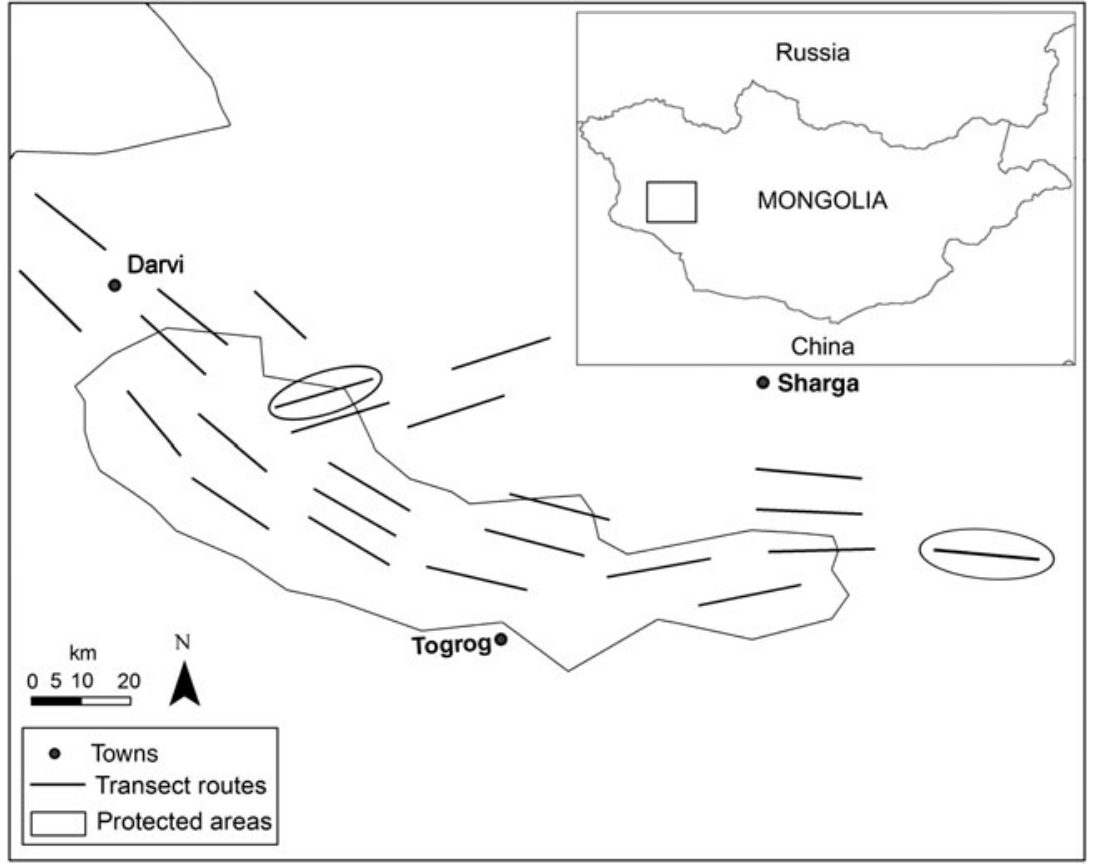

FIG. 1 The study area in and around Sharga Nature Reserve. Black lines illustrate the 24 transects used for distance sampling of Mongolian saiga Saiga tatarica mongolica in September 2006 and 2007. The two transects completed only in 2007 are circled. The square on the inset indicates the location of the main figure in western Mongolia.
Data were analysed using Distance v. 5 (Thomas et al., 2010). The variance of the encounter rate was estimated empirically using the replicate transect lines as samples, and maximum likelihood methods were used to estimate the variance of the effective strip width. There may be a tendency for smaller saiga groups to be missed more often than larger groups at further distances from the transect line, which can lead to size bias if the average group size $\bar{S}$ is used (Buckland et al., 2001). To test for bias in the estimate of group size we applied a statistical hypothesis test at the $15 \% \alpha$-level to the regression of natural logarithm of group size against the probability of detection at distance $x$ from the line, using Distance. If the regression is statistically significant, the expected group size $[E(s)]$ is used, otherwise average group size $(\bar{S})$ is used to estimate population density and size.

We first conducted exploratory analyses to examine options for truncation and grouping intervals to improve model fit for the detection function. Following Buckland et al. (2001) a variety of key functions and adjustment term combinations were considered to model the detection function (i.e. uniform + cosine or simple polynomial, half-

TABLE 1 Details of size of the study area (Fig. 1), number of transects, total effort $(L)$, and number of groups and individuals of Mongolian saiga Saiga tatarica mongolica observed in 2006 and 2007 .

\begin{tabular}{llllcl}
\hline Year & $\begin{array}{l}\text { Area } \\
\left(\mathrm{km}^{2}\right)\end{array}$ & $\begin{array}{l}\text { No. of } \\
\text { transects }\end{array}$ & $L(\mathrm{~km})$ & $\begin{array}{l}\text { No. } \\
\text { of groups }\end{array}$ & $\begin{array}{l}\text { No. of } \\
\text { individuals }\end{array}$ \\
\hline 2006 & 4,524 & 22 & 318.10 & 93 & 241 \\
2007 & 4,678 & 24 & 357.1 & 117 & 421 \\
\hline
\end{tabular}

normal + cosine or simple polynomial, hazard rate + cosine or hermite polynomial). Goodness-of-fit tests were used to identify violations of assumptions. Akaike's information criterion (Akaike, 1973) was used in model selection, with particular attention paid to model fit at distances near zero because this is important for robust estimation (Buckland et al., 2001).

\section{Results}

We observed 241 saiga in 93 groups in 2006 and 421 saiga in 121 groups in 2007 (Table 1). The survey area was smaller in 2006 because we were unable to complete two transects in that year (Fig. 1). Group sizes were 1-14 and 1-35 in 2006 and 2007, respectively (Table 2). For both years the size bias regression was significant $(\mathrm{P}<0.01)$, and thus the expected group size (Table 2) was used to estimate density. Encounter rate $(\mathrm{n} / L)$ was considerably lower in 2006 than in 2007 but with a similar percentage coefficient of variation (Table 3).

Saiga were typically far from observers when first detected $(577.7 \pm \mathrm{SE} 28.7 \mathrm{~m})$ but were detected as close as $80 \mathrm{~m}$. Detection and the effective strip width were estimated separately for each year (Table 4 ) because we had sufficient data per year. We also suspected that detectability varied between the 2 years because of differences in the amount of rainfall and resulting greenness of the vegetation: 2006 was dry and the vegetation was brown during the sampling periods, whereas 2007 was wet and green, resulting in a greater contrast between saiga coat colour and vegetation. In addition, although all observers were trained, the use of some different observers between years may have confounded comparisons. 
TABLE 2 Estimate of mean $\overline{\hat{s}}$ and expected group sizes $[E(\hat{S})]$ of Mongolian saiga in 2006 and 2007, with the 95\% confidence interval (95\% CI) and percentage coefficient of variation (\%CV) for the latter.

\begin{tabular}{lllll}
\hline Year & $\overline{\hat{s}}$ & $\hat{E}(S)$ & $95 \% \mathrm{CI}$ & $\% \mathrm{CV}$ \\
\hline 2006 & 2.47 & 1.90 & $2.018-3.028$ & 10.24 \\
2007 & 3.20 & 2.62 & $2.552-4.012$ & 11.45 \\
\hline
\end{tabular}

We inspected detailed histograms of detection frequencies by distance from the transect line to identify problems with the data (Figs 2a,c). In 2006 the histograms show too many observations close to zero and potential rounding at convenient values, which may be because of inaccurate angle measurements, especially at smaller angles (Fig. 2a). This tends to result in an effective strip width that is narrower than it should be, leading to a potential overestimate of abundance. In contrast, in 2007, the data indicate too many animals in the intervals slightly further from the line (Fig. 2c), suggesting animal movement away from the observers before the distance and angle measurements could be obtained. This tends to lead to an effective strip width that is wider than it should be and a potential underestimate of density and abundance.

To improve model fit the data were right truncated at $1,000 \mathrm{~m}$ for 2006 and $900 \mathrm{~m}$ for 2007 . In addition, to deal with the problem of animal movement and imprecision in the perpendicular distances resulting from inaccurate measurements, the data were grouped into equal-sized intervals (seven in 2006 and six in 2007). Considering Akaike's information criterion values and model fit close to zero for both years, a hazard rate model with no adjustment terms was selected to estimate detection probability (Figs $2 \mathrm{~b}, \mathrm{~d}$ ).

Estimates of saiga density were 0.54 and $0.78 \mathrm{~km}^{-2}$ in 2006 and 2007, respectively (Table 5) but were not statistically different between years $(z=0.98, \quad \mathrm{P}=0.33$; Buckland et al., 2001). Estimates of total abundance were 4,938 and 7,221 in 2006 and 2007, respectively (Table 5).

\section{Discussion}

Our population estimates for Mongolian saiga in the Sharga Nature Reserve and surrounding area are considerably larger

TABLE 3 The number of observed groups (n) after right truncation and the estimate of encounter rate $(\mathrm{n} / L)$ per $\mathrm{km}$ for Mongolian saiga in 2006 and 2007, with the $95 \%$ confidence interval (95\% CI) and percentage coefficient of variation (\%CV) for the latter.

\begin{tabular}{lrlll}
\hline Year & $\mathrm{n}$ & $\mathrm{n} / L\left(\mathrm{~km}^{-1}\right)$ & $95 \% \mathrm{CI}$ & $\% \mathrm{CV}$ \\
\hline 2006 & 89 & 0.280 & $0.180-0.436$ & 21.55 \\
2007 & 110 & 0.308 & $0.196-0.485$ & 22.18 \\
\hline
\end{tabular}

TABle 4 Estimate of $\hat{f}(\mathrm{o})$ and effective strip width $(\hat{\mu})$ for the Mongolian saiga surveys in 2006 and 2007, with the $95 \%$ confidence intervals ( $95 \% \mathrm{CI}$ ) and percentage coefficient of variation $(\% \mathrm{CV})$ for both $\hat{f}(0)$ and $\hat{\mu}$.

\begin{tabular}{lllllr}
\hline Year & $\hat{f}(0)_{\left(m^{-1}\right)}$ & $95 \%$ CI & $\hat{\mu}_{(m)}$ & $95 \%$ CI & $\% \mathrm{CV}$ \\
\hline 2006 & 0.0020 & $0.0014-0.0029$ & 495.90 & $342.68-717.62$ & 18.76 \\
2007 & 0.0019 & $0.0017-0.0023$ & 514.55 & $442.11-598.85$ & 7.67 \\
\hline
\end{tabular}

than previous estimates, based on minimum counts, of the entire Mongolian saiga population (Lushchekina et al., 1999; Amgalan et al., 2008). Our results suggest that, with adequate protection, sufficient numbers exist to facilitate in situ population recovery. To date, the international conservation strategy for saiga has often been based around an apparent need to establish a captive herd and breeding programme. Captive breeding of Mongolian saiga was identified as a priority within the CMS Medium Term Work Programme (CMS, 2006). However, this need was identified based on minimum counts that estimated only c. 1,800 saiga remained in the wild (Amgalan et al., 2008).

Our estimates were conducted in the autumn so that saiga would be widely distributed in small groups, to facilitate distance sampling, and at this time the observable population was probably at its highest for the year. Saiga, especially calves, may suffer high levels of mortality during periodically harsh Mongolian winters, known as $d z u d s$ (Bekenov et al., 1998). Previous counts were conducted in January (Amgalan et al., 2008), and differences between our results and those of Amgalan et al. (2008) may reflect overwinter mortality. Yet, no $d z u d s$ were reported during the sampling years for either study. Rather, it is likely that previous survey techniques resulted in underestimates of saiga populations. Underestimates of population size based on minimum counts probably resulted from the failure to estimate and correct for detection probability, as well as the exclusion of potentially suitable habitat for saiga from the survey area (e.g. minimum counts excluded canyons, to increase efficiency; Amgalan et al., 2008). Similarly, line transect estimates of ecologically equivalent North American pronghorn Antilocapra americana that did not account for detectability underestimated population abundance (Pojar \& Guenzel, 1999).

Although our estimates are substantially larger than minimum counts (Lushchekina et al., 1999; Amgalan et al., 2008), it is unclear whether the Mongolian saiga population is increasing. Saiga move according to vegetation, water and climactic conditions. Because these conditions may vary between years it is possible that the higher density in 2007 was a result of saiga movement into our study area rather than an actual increase in the population. In addition, two estimates of population size are insufficient to evaluate long-term trends. 

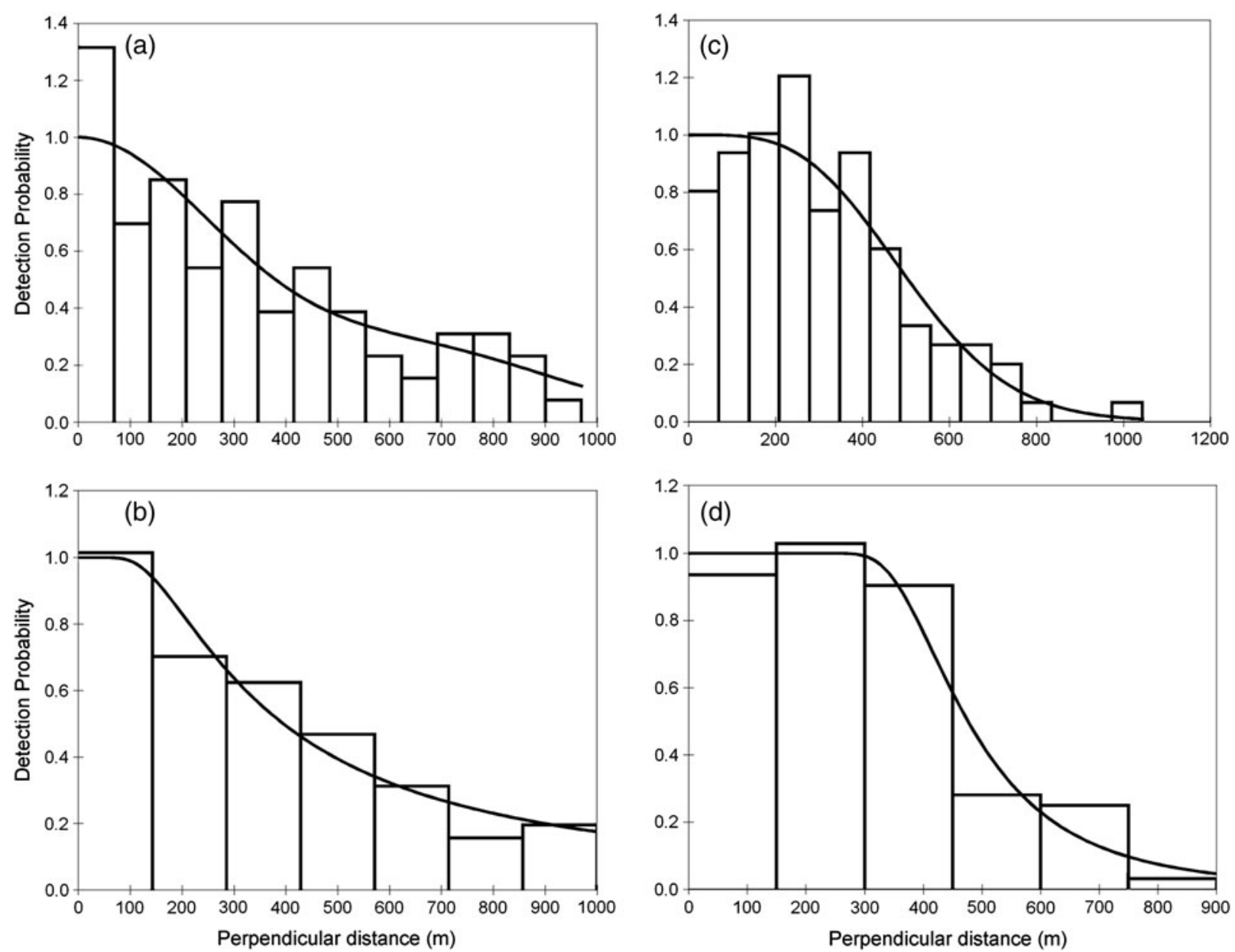

FIG. 2 Distance sampling data for Mongolian saiga with 5\% of the larger observations truncated for 2006 (a) and 2007 (c). In 2006 there is heaping at zero and possible rounding at other distances. In 2007 there is potential movement before the distance measurements were obtained. (b) and (d) illustrate, for 2006 and 2007 respectively, the detection function for the hazard rate model, with no adjustment terms, fitted to the perpendicular distances of observations of saiga groups. Data were grouped for final analysis using seven and six equal-spaced intervals in 2006 and 2007, respectively (with truncation at 1,000 m in 2006 and $900 \mathrm{~m}$ in 2007).

Population size is an important predictor of population persistence (Berger, 1990; Shaffer et al., 2000; Reed et al., 2003), although human threats can have profound and unpredictable impacts even on large populations (Ceballos \& Ehrlich, 2002; Altizer et al., 2003). For saiga, overharvesting and poaching resulted in their rapid and dramatic population decline (Milner-Gulland et al., 2001). Yet, as with pronghorn in North America (Byers \& Moodie, 1990; Byers, 1997), saiga demonstrate great recovery potential because females exhibit high fecundity and regular rates

TABLE 5 Estimates of density $(\hat{D})$ and abundance $(\hat{N})$ of Mongolian saiga in 2006 and 2007, with their 95\% confidence intervals $(95 \% \mathrm{CI})$ and the percentage coefficient of variation $(\% \mathrm{CV})$ for both $(\hat{D})$ and $(\hat{N})$.

\begin{tabular}{llllll}
\hline Year & $\hat{D}_{\left(\mathrm{Km}^{-1}\right)}$ & $95 \% \mathrm{CI}$ & $\hat{N}$ & $95 \% \mathrm{CI}$ & $\% \mathrm{CV}$ \\
\hline 2006 & 0.5366 & $0.300-0.959$ & 4,938 & $2,762-8,828$ & 29.73 \\
2007 & 0.7847 & $0.476-1.294$ & 7,221 & $4,380-11,903$ & 25.05 \\
\hline
\end{tabular}

of twinning (Kühl et al., 2007). In addition, c. 90\% of female saiga may reproduce within their first year (Fadeev \& Sludskii, 1982). Our population estimates, together with saiga reproductive characteristics, suggest that Mongolian saiga numbers are sufficient to withstand natural fluctuations caused by $d z u d s$. In addition, with adequate protection from poaching, and habitat management to ensure the availability of forage during winter (Berger et al., 2008), saiga numbers appear adequate to allow the population to remain at its historical population size $(>5,000$; Lushchekina et al., 1999).

Improving the precision of population estimates is a prerequisite for evaluating the effectiveness of conservation measures, as wide confidence intervals complicate detection of any trends. Such evaluations also depend on the magnitude of fluctuations in population size because of factors such as disease and weather relative to the impact of conservation actions such as anti-poaching measures. Because of constraints that limited survey effort, our 
population estimates had high variances, making it impossible to determine whether conservation efforts could have resulted in differences in population size between the 2 years. Similarly, large confidence intervals prevented detection of changes in population size of a threatened wallaby Onychogalea fraenata (Fisher et al., 2000). Because it is notoriously difficult to detect changes in population abundance of small and widely distributed populations, an important next step in developing a standardized monitoring protocol is to identify methods that improve precision, thereby increasing our ability to detect changes that result from a reduction in threats.

We have identified three modifications to our techniques that could reduce variance in distance sampling surveys. Firstly, accuracy and precision in data collection could be increased. Because saiga are chased with vehicles by poachers and by people wanting to see saiga, they typically flee from vehicles as far as $1 \mathrm{~km}$ distant. To determine preflight saiga locations we had to spot saiga and measure the radial distance $(r)$ from long distances. While our rangefinders were of high quality our compasses only measured to the nearest $2^{\circ}$. For example, one third of our sighting distances were $\geq 644 \mathrm{~m}$. If we observed a group of saiga at $644 \mathrm{~m}$ and $60^{\circ}$, but the animal was actually at $644 \mathrm{~m}$ and $64^{\circ}$, then we would calculate the group's perpendicular distance $21 \mathrm{~m}$ too far from its actual distance to the transect line. We therefore recommend the use of compasses that provide more accurate sighting angles.

Secondly, to reduce problems associated with the fleeing behaviour of saiga alternative modes of transportation could be tested. Eliminating the use of vehicles may decrease distances to which observers can approach and obtain original locations. We observed saiga near livestock, especially camels and horses, grazing near human settlements and gers (Mongolian nomadic home). If saiga are tolerant of livestock, distance sampling by camel or horseback could improve ability to detect saiga at their original locations and thus obtain more accurate measurements. However, this may not be appropriate for a small team conducting range-wide surveys because of the substantial time and associated logistics required. Additional teams and training would be needed to complete such a range-wide survey, and associated increase in costs could be prohibitive. This technique may, however, be useful for rugged areas where travel by vehicle impedes the ability to detect saiga. We identified a small and rugged area that includes c. $1,056 \mathrm{~km}^{2}$ of Mongolian saiga range in which a pilot survey could evaluate the feasibility of using horses or camels. Another option is aerial surveys, which would facilitate distance sampling of the entire Mongolian saiga range $\left(\right.$ c. $\left.6,900 \mathrm{~km}^{2}\right)$. Aerial surveys have been conducted for distance sampling of large mammals in southern Mongolia (Reading et al., 1999) but, to our knowledge, there are currently no planes or helicopters in Mongolia that could fly at the slow speeds required and there is no national plan to facilitate the use of planes from other range states. More information is needed to determine if the logistical costs and potential disadvantages (imperfect detection on the line because of the type or speed of the aircraft) of an aerial survey would outweigh any benefits. Although Norton-Griffiths \& McConville (2007) evaluated survey techniques for saiga in Kazakhstan the differences in biology, habitat and population sizes between the two saiga subspecies may preclude useful comparisons of the suitability of techniques to the two areas.

Thirdly, we conducted a power analysis using Trends $v$. 3.o (Gerrodette, 1993), setting the $\% \mathrm{CV}$ at $10 \%$, a value recently determined as acceptable for population estimates of Mongolian saiga by the Mongolian Academy of Sciences (L. Amgalan, pers. comm.). We calculated the sampling effort needed to produce estimates with the precision required for monitoring of population trends (Buckland et al., 2001). We based our parameter values for encounter rate and variance in group size on the results of this study and determined that sampling should include at least $1,500 \mathrm{~km}$ of effort, assuming an encounter rate of c. 0.3 saiga $\mathrm{km}^{-1}$. If the encounter rate were to increase to 0.5 saiga $\mathrm{km}^{-1}$, then $\mathrm{c}$. $900 \mathrm{~km}$ of effort would be sufficient. This required level of effort is not definitive, however. Our sampling efforts were 318.1 and $357.1 \mathrm{~km}$ in 2006 and 2007 , respectively, giving a $\% \mathrm{CV}$ of 30 and $25 \%$, respectively, and our survey design was based on the best information available at the time. Only after using a modified design will it become evident if our recommended changes are sufficient or if further modifications are still required.

Although variance needs to be reduced and the field protocol for distance sampling improved, our results demonstrate that distance sampling can be an effective technique for monitoring saiga populations in Mongolia. Distance sampling has the potential to produce unbiased absolute values of abundance. It is a practical method that is non-invasive and comparatively inexpensive for large geographical areas (Fisher et al., 2000). Successful application to Mongolian saiga suggests that it could be used to determine population size and assess impacts of natural and human-induced threats on saiga populations throughout the range states. The methodology's flexibility allows for the differences in landscape and species characteristics across the range states. For example, the area that saiga occupy in some range states, such as Kazakhstan and Uzbekistan, are vast compared to the range of Mongolian saiga. The mode of transport (i.e. aerial vs terrestrial) can be adapted to take account of this difference. The population and group size of Mongolian saiga are small compared to saiga in Kazakhstan and Russia and distance sampling can be applied to a range of population sizes. However, group sizes in the hundreds and thousands are common in 
Kazakhstan, which would make distance sampling harder to apply. Pilot studies are necessary in other range states to compare distance sampling to alternative sampling methods. We suggest that signatories of the MOU (CMS, 2006) consider adopting distance sampling as the standardized monitoring protocol for regular survey of saiga populations.

\section{Acknowledgements}

We are indebted to L. Amgalan, B. Lkhagvasuren, A. Fine and P. Zahler for assistance with this project. We thank staff at the Institute of Biology of the Mongolian Academy of Sciences and at the Wildlife Conservation Society, Mongolia Country Program Office for their logistical support. D. Chin-Unen and many others assisted with fieldwork and at the camp. H. Weaver and B. Hudgens commented on earlier drafts of this article. The project was funded by a grant from the National Geographic Society.

\section{References}

A kaike, H. (1973) Information theory and an extension of the maximum likelihood principle. In Second Symposium on Information Theory (eds B.N. Petran \& C.S. Bsaki), pp. 267-281. Akademia Kiadz, Budapest, Hungary.

Altizer, S., Harvell, D. \& Friedle, E. (2003) Rapid evolutionary dynamics and disease threats to biodiversity. Trends in Ecology \& Evolution, 18, 589-596.

Amgalan, L., Buuveibatiar, B. \& Chimeddorj, B. (2008) Current distribution and number of the Mongolian saiga (Saiga tatarica mongolica). Journal of Institute of Biology, 27, 4-6. [in Mongolian]

Bannikov, A.G., Zhirnov, L.V., Lebedeva, L.S. \& Fandeev, A.A. (1961) Biology of saiga. Selskokchozyajstvennaya Literature Publications, Moscow, Russia. [in Russian]

Bekenov, A.B., Grachev, I.A. \& Milner-Gulland, E.J. (1998) The ecology and management of the saiga antelope in Kazakhstan. Mammalian Review, 28, 1-52.

Berger, J. (1990) Persistence of different-sized populations: an empirical assessment of rapid extinctions in bighorn sheep. Conservation Biology, 4, 91-98.

Berger, J., Young, J.K. \& Berger, K.M. (2008) Protecting migration corridors: challenges and optimism for Mongolian saiga. PLoS Biology, 6, 1365-1367.

Biswas, S. \& Sankar, K. (2002) Prey abundance and food habit of tigers (Panthera tigris tigris) in Pench National Park, Madhya Pradesh, India. Journal of Zoology, London, 256, 411-420.

Buckland, S.T., Anderson, D.R., Burnham, K.P., LaAke, J.L., Borchers, D.L. \& Thomas, L. (2001) Introduction to Distance Sampling: Estimating Abundance of Biological Populations. Oxford University Press, Oxford, UK.

Byers, J.A. (1997) American Pronghorn: Social Adaptations and the Ghosts of Predators Past. University of Chicago Press, Chicago, USA.

Byers, J.A. \& Moodie, J.D. (1990) Sex-specific maternal investment in pronghorn, and question of a limit on differential provisioning in ungulates. Behavioral Ecology and Sociobiology, 26, 157-164.

Ceballos, G. \& Ehrlich, P.R. (2002) Mammal population losses and the extinction crisis. Science, 296, 904-907.
Chimeddorj, B., Amgalan, L. \& Buuveibaatar, B. (2009) Current status and distribution of the saiga in Mongolia. Saiga News, 8, 1.

Clark, E.L. \& Javzansuren, M. (2006) Mongolian Red List of Mammals. Zoological Society of London, London, UK.

CMS (Convention of Migratory Species) (2006) Medium Term International Work Programme for the Saiga Antelope (2007-2011). CMS/SA-1/Report Annex 9. Http://www.cms.int/species/saiga/ post_session/Annex_09_MediumTerm_Int_WrkProgm_E.pdf [accessed 1 April 2009].

Fadeev, V.A. \& Sludskil, A.A. (1982) The Saiga in Kazakhstan. Academy of Sciences, Alma-Ata, Kazakhstan, USSR.

Fisher, D.O., Hoyle, S.D. \& Blomberg, S.P. (2000) Population dynamics and survival of an endangered wallaby: a comparison of four methods. Ecological Applications, 10, 901-910.

Focardi, S., Montanaro, P., Isotti, R., Ronchi, F., Scacco, M. \& Calmanti, R. (2005) Distance sampling effectively monitored a declining population of Italian roe deer Capreolus capreolus italicus. Oryx, 39, 421-428.

Gerrodette, T. (1993) Trends: software for a power analysis of linear regression. Wildlife Society Bulletin, 21, 515-516.

Kholodova, M.V., Lushchekina, A.A., Neronov, V.M., Strelkova, M.V., Niyambayar, N. \& Amgalan, L. (2001) Comparative analysis of the Kalmyk and Mongolian Saiga genetic diversity. Doklady Biological sciences, 376, 102-104.

Koenen, K.G., DeStefano, S. \& Krausman, P.R. (2002) Using distance sampling to estimate seasonal densities of desert mule deer in a semi-desert grassland. Wildlife Society Bulletin, 30, 53-63.

Kühl, A., Mysterud, A., Erdnenov, G.I., Lushchekina, A.A., Grachev, I.A., Bekenov, A.B., \& Milner-Gulland, E.J. (2007) The 'big spenders' of the steppe: sex specific maternal allocation and twinning in the saiga antelope. Proceedings of the Royal Society B, 274, 1293-1299.

Lushcherina, A.A., Dulamtseren, S., Amgalan, L. \& Neronov, V.M. (1999) The status and prospects for conservation of Mongolian saiga, Saiga tatarica mongolica. Oryx, 33, 21-30.

MALLON, D.P. (2008) Saiga tatarica mongolica. In IUCN Red List of Threatened Species v. 2009.2. Http://www.iucnredlist.org [accessed 20 January 2010].

Milner-Gulland, E.J., Kholodova, M.V., Bekenov, A.B., Bukreeva, O.M., Grachev, Iu.A., Amgalan, L. \& Lushcherina, A.A. (2001) Dramatic declines in saiga antelope populations. Oryx, 35, 340-345.

Norton-Griffiths, M. \& McConville, A.J. (2007) Republic of Kazakhstan. Technical Evaluation of the Aerial Survey Programme for Saiga Antelope (Saiga tatarica) with recommendations for improvements. FZS Saiga Aerial Census Final Report. Frankfurt, Germany.

Olson, K.A., Fuller, T.K., Schaller, G.B., Odonkhuu, D. \& Murray, M.G. (2005) Estimating the population density of Mongolian gazelles Procapra gutturosa by driving long distance line transects. Oryx, 39, 164-169.

Pojar, T.M. \& Guenzel, R.J. (1999) Comparison of fixed-wing line transect and helicopter quadrat pronghorn surveys. Proceedings of the Pronghorn Antelope Workshop, 18, 64-68.

Reading, R.P., Mix, H., Lhagvasuren, B. \& Blumer, E.S. (1999) Status of wild Bactrian camels and other large ungulates in southwestern Mongolia. Oryx, 33, 247-255.

Reed, D.H., O'Grady, J.J., Brook, B.W., Ballou, J.D. \& FrANKHAM, R. (2003) Estimates of minimum viable population sizes for vertebrates and factors influencing those estimates. Biological Conservation, 113, 23-34.

Schaller, G.B. (1998) Wildlife of the Tibetan Steppe. University of Chicago Press, Chicago, USA. 
Seddon, P.J., Ismail, K., Shobrak, M., Ostrowski, S. \& Magin, C. (2003) A comparison of derived population estimate, markresighting and distance sampling methods to determine the population size of a desert ungulate, the Arabian oryx. Oryx, 37, 286-294.

Shaffer, M.L., Hood, L., Snape, W.J.I. \& Latchis, I. (2000) Population viability analysis and conservation policy. In Population Viability Analysis (eds S.R. Beissinger \& D.R. McCullough), pp. 123-142. University of Chicago Press, Chicago, USA.

Thomas, L., Buckland, S.T., Rexstad, E.A., Laake, J.L., Strindberg, S., Hedley, S.L. et al. (2010) Distance software: design and analysis of distance sampling surveys for estimating population size. Journal of Applied Ecology, 47, 5-14.

Whit taker, D.G., Van Dyke, W.A. \& Love, S.L. (2003) Evaluation of aerial line transect for estimating pronghorn antelope abundance in low-density populations. Wildlife Society Bulletin, 31, $443-453$.

\section{Biographical sketches}

Julie K. Young is a spatial and behavioural ecologist. She has worked on conservation-based research projects in Asia, Africa, and South and North America. KIM M. MURRAY is a population ecologist and has studied caribou, moose, pronghorn and saiga antelope, and coyotes, wolves and snow leopards. Samantha STRINDBERg is a biostatistician focused on wildlife population assessment at Wildlife Conservation Society field sites. She has a particular interest in the design of distance sampling surveys and helped develop the automated survey design component of the software Distance. BAYARBAATAR BUUVEIBAatar was the recipient of a Zoological Society of London EDGE grant for his work on Mongolian saiga and spends many field hours researching Mongolia's threatened species. JOEL BERGER's research focuses on the conservation of species and intact ecosystems. 